\begin{abstract}
\begin{tabular}{llllllll} 
A & B & S & T & R & A & C & T \\
\hline
\end{tabular}
The association of risk factors such as smoking, nutrition and adolescence with low birthweight has been well documented. What is less known is the extent to which certain program interventions can mediate the impact of these risks on low birthweight and the level of intervention necessary to have an impact. This study had two objectives: (a) to explore the role of the Toronto Healthiest Babies Possible (HBP) program as a mediator of the impact of smoking, adolescence, prepregnancy underweight and erratic weight gain/initial weight loss on LBW, and (b) to determine the dose-response relationship between the number of home visits and LBW. Study results suggest that the HBP strategy is making a difference for clients with the aforementioned three risk factors, and that the most effective intervention "dose" to reduce low birthweight is 9-11 visits. This type of study may serve as a benchmark in the evaluation of other similar programs.
\end{abstract}

\begin{tabular}{llllll} 
A & B & R & E & G & É \\
\hline
\end{tabular}

L'association qui existe entre d'une part des facteurs de risque tels que le tabagisme, la nutrition et l'adolescence, et d'autre part le faible poids à la naissance est bien connue. Ce que l'on connaît moins c'est la mesure dans laquelle certaines interventions peuvent atténuer l'impact de ces risques sur le faible poids à la naissance et le niveau d'intervention nécessaire pour ce faire. Cette étude avait deux objectifs : (a) étudier le rôle du programme HBP (Healthiest Babies Possible) de Toronto en tant qu'élément pour atténuer l'impact du tabagisme, de l'adolescence, de l'insuffisance pondérale et de la prise de poids erratique/perte de poids initiale avant la grossesse sur les naissances de faible poids, et (b) déterminer la relation dose-réponse entre le nombre de visites à domicile et le faible poids à la naissance. Les résultats de l'étude semblent indiquer que la stratégie suivie par le programme HBP change les choses pour les clients concernés par les trois facteurs de risque susmentionnés, et que la "dose " d'intervention la plus efficace pour réduire le faible poids à la naissance est de l'ordre de 9 à 11 visites. Ce type d'étude pourra servir de référence à l'évaluation d'autres programmes analogues.

How Many Visits by Health Professionals are Needed to Make a Difference in Low Birthweight? A Dose-response Study of the Toronto Healthiest Babies Possible Program

\author{
Ellen Desjardins, MHSc, Deborah Hardwick, MA
}

Programs for the prevention of low birthweight (LBW) are a fundamental component of public health. A great deal of research has focused on identifying the main risk factors for LBW, their degree of influence on LBW, and the interactions between them. ${ }^{1,2}$ Socio-economic conditions $^{3-5}$ maternal nutrition ${ }^{6-8}$ adolescence ${ }^{9}$ and smoking ${ }^{10,11}$ are strongly associated with LBW. What is also of interest, however, is the extent to which certain health program strategies can mediate the impact of these risks on LBW and the level of intervention necessary to have an impact.

Several programs which aim to reduce low birthweight currently exist in Canada. ${ }^{12}$ Those which involve one-to-one counselling to socio-economically disadvantaged pregnant women do so either as home visits or at a central location, and usually also provide supplemental food or milk. One-to-one counselling allows the variety of individual needs to be addressed effectively. Food supplements serve as an incentive to join, a nutritional boost and an aid to dietary change. This combined strategy has been demonstrated at some sites to have a positive effect on birthweight. ${ }^{13-15}$ A source of variation, however, is the type and combination of staff who implement the program: most common are dietitians, public health nurses, social

\section{Toronto Public Health}

Correspondence: Ellen Desjardins, 146 Briarcliffe Cres., Waterloo, ON, N2L 5T8, Tel: 519-883-2002, ext 5166, Fax: 519-883-2241, E-mail: dellen@region.waterloo.ca

Reprint requests: Deborah Hardwick, 277 Victoria St., 6th Floor, Toronto, ON, M5B 1W2, Tel: 416392-7451, Fax: 416-392-1483, E-mail: dhardwic@city.toronto.on.ca workers and peer health workers. Several studies have looked at prenatal home visiting programs without the food supplement component; although these did not make a significant impact on low birthweight, other positive outcomes were found. ${ }^{16-19}$

It is difficult to compare the effectiveness of these programs solely by their overall incidence of low birthweight because of the differences in the type and intensity of intervention. It is also methodologically and ethically problematic for programs with only a few hundred participants per year to compare their birth outcomes with a matched, equally disadvantaged group who did not attend. The Montreal Diet Dispensary has been able to use some unique comparison groups such as siblings, due to their very large numbers of contacts. ${ }^{13}$ The within-mother efficacy analysis showed that the impact of the Higgins Nutrition Intervention Program ${ }^{13}$ was significantly greater for the group with a minimum of four dietitian-client contacts during the pregnancy, compared to a nonintervention group and the group that had any number of dietitian-client contacts.

In this paper we examine birthweight outcome among clients enrolled in the Healthiest Babies Possible (HBP) program in Toronto. HBP has provided home counselling for pregnant women by Toronto public health dietitians and nurses in equal proportion since 1979 . Coupons for 1 litre of milk per day are provided to clients. A high risk, low income population is reached. In addition to low income, $52 \%$ were assessed as having 4-6 risk factors, and an additional $20 \%$ had 7 or more risk factors. ${ }^{20}$ The content of counselling 
sessions varies according to individual client needs, risks and strengths; it is usually a combination of educational and supportive components. Dietitians' sessions almost always involve a 24-hour recall for assessment and planning purposes. For this study, we combined data for the years 1987 to 1996 , during which time the program remained consistent. Variables routinely measured during this time were maternal adolescence, pre-pregnancy underweight status, smoking and initial weight gain. These variables are all associated with preterm and/or SGA (small for gestational age) births. ${ }^{1}$

In this study, we also explore the relationships between LBW and selected risk factors for different levels of intervention (number of visits). This would be helpful to know for two reasons: 1) to provide information which might help maximize effectiveness of this program, and 2) to serve as a potential benchmark for comparison of similar programs with variations such as types of staff or counselling locations. Individual counselling and food supplements are resource-intensive interventions; therefore it is prudent to suggest program models that are cost effective because they reduce low birthweight with a minimum number of visits.

The epidemiological method of "dose response" is used to find a relationship between levels of exposure to a risk and incidence of disease. ${ }^{21} \mathrm{We}$ adopted this concept to study the relationship of the levels of exposure to an intervention and the incidence of LBW. This methodology has been used to study the relationship between the number of home visits and post-natal outcomes for low birthweight babies. ${ }^{22} \mathrm{~A}$ dose-response relationship will enable us to hypothesize the most effective number of visits required to reduce the probability of low birthweight.

This study had two objectives: 1) to explore the role of HBP as a mediator of the impact of smoking, adolescence, prepregnancy underweight and erratic weight gain/initial weight loss on LBW, and 2) to determine the dose-response relationship between the number of home visits and LBW.

\section{METHODS}

\section{Study population}

The population studied were the women registered in HBP from January 1, 1987 to December 31, 1996. During this time, 2,956 women entered the HBP program, of whom 2,046 (69\%) remained in the program at least until the birth of their babies.

Twenty-one percent of all HBP clients were aged 18 years or younger, $29 \%$ were smokers, $19 \%$ were initially underweight and $37 \%$ had inadequate weight gain.

\section{Sample}

This analysis is restricted only to HBP clients who completed the program to delivery. About $31 \%$ did not complete the program for a variety of reasons (most often because they moved out of city limits), and outcome information is not available for most of them. Bivariate analysis* indicate that clients initially assessed with erratic or inadequate weight gain were about 1.5 times more likely to complete the program. Adolescents and smokers were less likely to complete the program. Those who were underweight prior to becoming pregnant were no more or less likely than those without this risk factor to complete the program.

Only singleton births are included in this study because of the strong relationship between multiple births and low birthweight. We further excluded those clients for whom the number of visits was missing, since this is our main predictor variable. The resulting sample size is 1,883 , or $64 \%$ of all women initially registered in HBP during the study period.

\section{Variables}

Client data forms were completed by dietitians and nurses for each client, from intake to discharge. The outcome variable, low birthweight, is derived from the baby's birthweight as reported by the mother on discharge from the HBP program. This figure was confirmed by birth registration

\footnotetext{
* Odds ratios of completing the program: Inadequate or erratic weight gain - 1.55 $(95 \%$ $\mathrm{CI}=1.31-1.84), \mathrm{p}=0.00$; Adolescence -0.73 (95\% CI $=0.60-0.88), \mathrm{p}=0.01$; Smoking -0.1 $(95 \% \mathrm{CI}=0.67-0.98), \mathrm{p}=0.2$
}

forms sent to the health unit for those who gave birth in the City of Toronto. It is a dichotomous variable, with birthweights $2500 \mathrm{~g}$ and more coded as 0 , and low birthweight (less than $2500 \mathrm{~g}$ ) coded as 1 for the logistic regression analysis. There were 128 singleton births with birthweight less than 2500 grams.

The predictor variable, number of visits, was a continuous variable that ranged from 1 to 26 , as reported by the dietitians and nurses upon discharge. For the final three logistic regression models, the variable was trichotomized as described in the data analysis section below. Each level (low, medium, high) was coded as 1 and entered individually into separate models.

The initial risk factors - smoking, adolescence, inadequate weight gain and prepregnancy underweight - were determined by the dietitian or nurse at intake. The client was recorded as smoking if she reported smoking any number of cigarettes. Five hundred and forty-eight (548) clients reported smoking. There were 362 adolescents in the study (i.e., those clients aged 18 or younger at registration in the program). Inadequate weight gain was recorded as a risk factor if the client was assessed by the dietitian as having weight loss, lack of weight gain or very inadequate weight gain since conception. Seven hundred and fifty-five (755) clients had this risk factor. Prepregnancy weight is self-reported, but dietitians bring weigh scales to every home visit. Prepregnancy BMI was calculated for those 19 years of age and older, and those with a BMI less than 20 were recorded as being underweight. For adolescents, weight status was determined by the dietitian's assessment. In total, 388 clients in the sample were underweight.

For multivariate analysis, these variables were coded 1 if the risk factor was present and 0 if absent.

\section{Data analysis}

Bivariate analyses were completed for the predictor and outcome variables (low birthweight by number of visits) and also for initial risk factors (smoking, adolescence, inadequate weight gain, and prepregnancy underweight) by low birthweight and by number of visits. 


\begin{tabular}{|c|c|c|c|c|c|}
\hline \multicolumn{6}{|c|}{ Incidence of LBW Among } \\
\hline & \multicolumn{2}{|c|}{ N (\% of sample) } & LBW (\%) & \multicolumn{2}{|c|}{ Odds Ratio $(95 \% \mathrm{Cl}) \dagger$} \\
\hline $\begin{array}{l}\text { Total Sample } \\
\text { Risk Factors }\end{array}$ & 1892 & $(100)$ & $128 \quad(6.8)$ & $\mathrm{N} / \mathrm{A}$ & \\
\hline Adolescence & 362 & (19.1) & (8.3) & 1.29 & $(0.82-2.02)$ \\
\hline Smoking & 548 & (29.0) & $(9.1)$ & $1.65^{*}$ & $(1.11-2.46)$ \\
\hline Underweight & 388 & (20.5) & (8.8) & 1.43 & $(0.93-2.20)$ \\
\hline Inadequate weight gain & 755 & (39.9) & $55 \quad(7.3)$ & 1.15 & $(0.78-1.67)$ \\
\hline
\end{tabular}

\begin{tabular}{|c|c|c|c|c|}
\hline \multicolumn{5}{|c|}{$\begin{array}{l}\text { TABLE II } \\
\text { Birthweight Outcome Among HBP Clients by Number of Visits }\end{array}$} \\
\hline & $\begin{array}{l}\text { Low } \\
(<9)\end{array}$ & $\begin{array}{l}\text { Visits } \\
\text { Medium } \\
(9-11)\end{array}$ & $\begin{array}{c}\text { High } \\
\text { (12 or more) }\end{array}$ & Total \\
\hline $\begin{array}{l}\text { Birthweight Outcome } \\
<2500 \text { g (low birthweight) } \\
2500 \text { g or more } \\
\text { Total }\end{array}$ & $\begin{aligned} 70 & (8.8 \%) \\
723 & (91.2 \%) \\
793 & \end{aligned}$ & $\begin{aligned} 27 & (5.1 \%) \\
501 & (94.9 \%) \\
528 & \end{aligned}$ & $\begin{aligned} 31 & (5.5 \%) \\
531 & (94.5 \%) \\
562 & \end{aligned}$ & $\begin{array}{r}128(6.8 \%) \\
1755 \\
1883\end{array}$ \\
\hline
\end{tabular}

Logistic regression models were run for total number of visits (as a continuous variable) with the initial risk factors. The selected risk factors were included in the multivariate analyses to explore their possible influence on the effect that number of visits had on low birthweight.

Total number of visits was reconceptualized as a trichotomous variable to test the dose-response relationship between number of visits. This was an attempt to identify the most effective number of visits. Clients receiving 1-8 visits were categorized as having "low" visits, those receiving 9-11 were "medium" and 12 or more were "high". These cut-offs were selected because approximately one third of the sample fell within those categories. The breakdown was: 793 "low", 528 "medium" and 562 "high". Logistic regression models were then run for each level of visit, with the selected risk factors.

Odds ratios were calculated for the bivariate and multivariate analyses and were accepted as significant if the chisquare probability was 0.05 or less.

\section{RESULTS}

Table I shows that, regardless of the number of visits, the program appears to be making a difference for all selected risk factors in this study except for smoking. Among all mothers in this study, 6.8\% had low birthweight babies. After having par- ticipated in the HBP program, mothers who had initial risk factors other than smoking showed no statistically significant difference in LBW compared to those who did not. However, there is a statistically significant difference between smokers and non-smokers. Among smokers, 9.1\% $(\mathrm{n}=50)$ had low birthweight babies, compared to $5.7 \%(\mathrm{n}=66)$ among non-smokers. Smokers were over $60 \%$ more likely to have low birthweight babies than nonsmokers, in spite of their participation in the program.

The total number of visits ranged from 1 to 26 , with a median of 9 . About $42 \%$ $(\mathrm{n}=793)$ clients received 1-8 visits, 28\% received 9-11 visits and 30\% received 12 visits or more. Of those in the low visit group, $8.8 \%(\mathrm{n}=70)$ had LBW babies, compared to $5.1 \%(\mathrm{n}=27)$ in the medium and $5.5 \%(n=31)$ in the high visit groups. When comparing low birthweight based only on the number of visits, the relationship between these variables is statistically significant, as shown in Table II.

The results of the logistic regression analysis are summarized in Table III. The number of visits by dietitians and public health nurses, when entered as a continuous variable, independently reduces the odds of low birthweight by $10 \%$ for each visit (Model 1). Model 2 introduces gestational age and smoking to the logistic regression. However, these two variables have minimal impact on the relationship between number of visits and low birthweight and are, themselves, not related to low birthweight.

When the initial risk factors are taken into account, the effect of the total number of visits changes very little, indicating a stable and negative relationship between number of visits and LBW (Model 3). In this model, none of the risk factors are associated with LBW.

In the logistic regression models that include low, medium or high number of visits as the predictor variable, clients who receive low visits have a $70 \%$ greater probability of having LBW babies than those who receive 9 visits or more, while controlling for the selected risk factors (Model 4). On the other hand, those who receive the medium number of visits (9-11) may be $35 \%$ less likely to have LBW babies than those who receive lower or higher numbers of visits (Model 5). The odds ratio for Model 4 is statistically significant, and Model 5 is almost statistically significant $(\mathrm{p}=0.057)$. The odds ratio is not statistically significant for those who receive 12 visits or more.

\section{DISCUSSION}

Between 1987 and 1996, the mothers who stayed in HBP until the birth of their babies and who were adolescent, underweight or had erratic weight gain or initial weight loss appeared to benefit from the program in terms of LBW, regardless of the number of visits. The overall LBW was $6.8 \%$, compared to about $5.2 \%$ of singleton births in the City of Toronto during the same period. The City's LBW figure includes women of all ages and risk factors, whereas the HBP clients all have low income and at least one additional risk factor. While greater percentages of mothers with the risk factors we studied had LBW babies compared to mothers without those risk factors, the differences were not statistically significant. Mothers who smoke were still more likely to have LBW babies than those who did not. However, studies of expectant mothers in other populations indicate that those who smoke are about twice as likely to have a low birthweight baby as non-smokers. ${ }^{23}$ This suggests that HBP Toronto may be effective in reducing 


\section{TABLE III}

\section{Logistic Regression of LBW on Number of Visits and Selected Risk Factors}

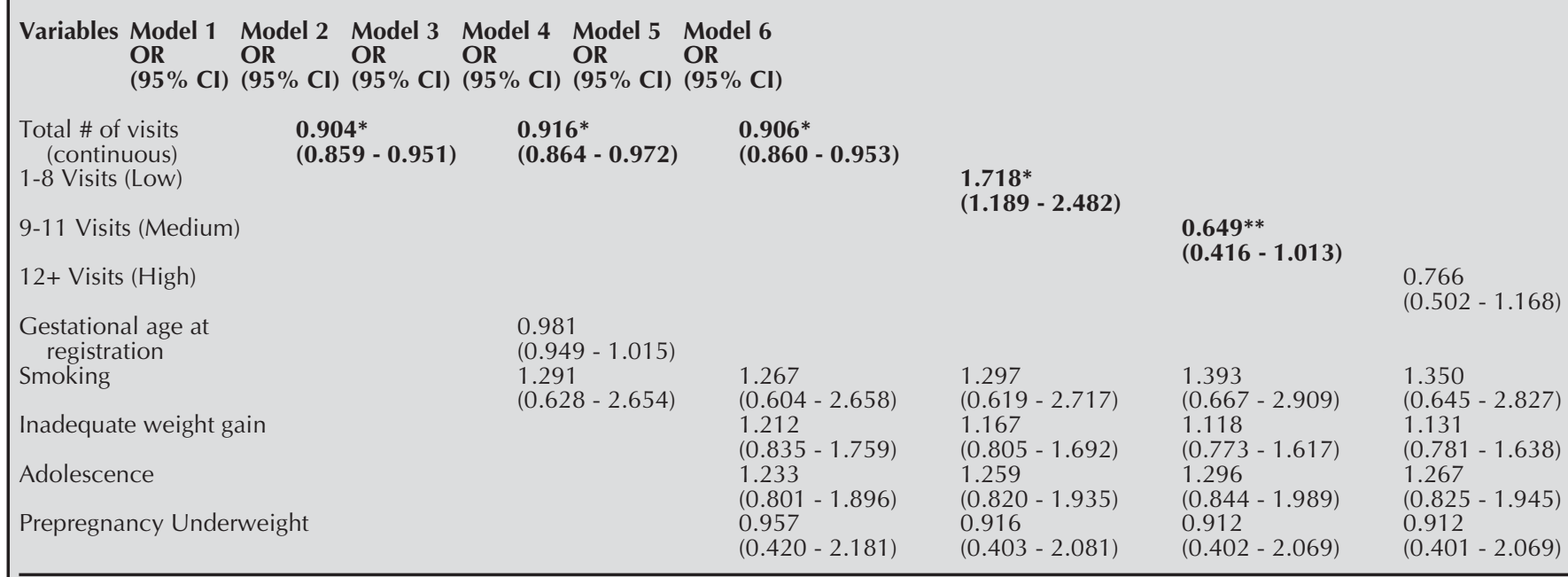

* $\mathrm{p} \leq 0.05$

** $p=0.057$

the number of LBW babies among smokers, since smoking clients are only $60 \%$ more likely to have low birthweight babies.

By conceptualizing the number of visits as either low, medium or high doses of intervention, we are given a more realistic picture of the association between the number of visits and LBW, than if we keep the number of visits as a continuous variable. We found that the probability of LBW decreased by about $10 \%$ for each HBP visit when using the continuous variable. However, this does not tell us whether each visit has equal effect. In other words, it does not tell us if there is any threshold or best number of visits. By categorizing the number of visits into low, medium and high, the findings suggest that there is a dose-response relationship between HBP home visits and LBW and that the best "dose" appeared to be about 9-11 visits. The percentage of clients who received 9-11 visits and had low birthweight babies (5.1\%) was similar to that of the City of Toronto as a whole $(5.2 \%)$, whereas $8.8 \%$ of those who received fewer than 9 visits had low birthweight babies.

This suggests that one-to-one counselling by public health nurses and dietitians, along with provision of milk coupons, approximately every 2 to 3 weeks during the last two trimesters of pregnancy may be sufficient to make a significant difference in low birthweight. Fewer visits, as a result of starting the program later in pregnancy or having visits further apart, may be ineffectual in reducing the risk of LBW. However, more than 12 visits may not provide continuously better results in terms of low birthweight. If the HBP program had been restricted to providing the lowest level of intervention (1-8 visits), the probability of LBW would have been $70 \%$ higher. This means an additional 90 low birthweight babies would have been born over the 10-year period of this study.

The results of this study were achieved with one-to-one counselling in client's homes, using professional dietitians and public health nurses, the provision of milk coupons, and a caseload which has always been low income, multicultural and with a majority of multiple-risk clients. These results may, however, be inconclusive given the broad confidence intervals of the odds ratios in the logistic regression models. Therefore, further research with a prospective design needs to be completed to confirm the most effective number of prenatal home visits required to reduce the risk of LBW among high-risk women. Future research should also explore the dose-response relationship between number of visits and low birthweight for outof-the home counselling, lay home visiting, different types of food supplements, or other variations in program implementation, as well as for populations with a different demographic profile.

\section{ACKNOWLEDGEMENTS}

The authors thank Fred Goettler and Carol Paulsen, Health Information and Research Services, Toronto Public Health, for their technical advice and support.

\section{REFERENCES}

1. Lang JM, Lieberman E, Cohen A. A comparison of risk factors for preterm labor and term smallfor-gestational-age birth. Epidemiology 1996;7:369-76.

2. Cogswell ME, Yip R. The influence of fetal and maternal factors on the distribution of birthweight. Semin Perinatol 1995;19(3):222-40.

3. Rutter DR, Quine L. Inequalities in pregnancy outcome: A review of psycho-social and behavioural mediators. Soc Sci Med 1990;30(5):55368.

4. Turner RJ, Grindstaff CF, Phillips N. Social support and outcome in teenage pregnancy. $J$ Health Soc Behav 1990; 31:43-57.

5. Wilcox MA, Smith SJ, Johnson IR, et al. The effect of social deprivation on birthweight, excluding physiological and pathological effect. Br J Obstet Gynaecol 1995;102:918-24.

6. Abrams B, Selvin S. Maternal weight gain pattern and birth weight. Obstet Gynecol 1995;86:16369.

7. Moutquin JM, Milot-Roy V, Irion O. Preterm birth prevention: Effectiveness of current strategies. J SOGC 1996;18:571-88.

8. Doyle W, Crawford MA, Wynn AHA, Wynn SW. Maternal nutrient intake and birthweight. J Hum Nutr Diet 1989;2:415-22.

9. Dubois S, Coulombe C, Pencharz P, et al. Ability of the Higgins Nutrition Intervention Program to improve adolescent pregnancy outcome. J Am Diet Assoc 1997;97:871-78.

10. Muscati SK, Gray-Donald K, Newson EE. Interaction of smoking and maternal weight status in influencing infant size. Can J Public Health 1994;85(6):407-12.

11. Shu XO, Hatch MC, Mills J, et al. Maternal smoking, alcohol drinking, caffeine consumption 
and fetal growth: Results from a prospective study. Epidemiology 1995;6:115-20.

12. Desrosiers Choquette J, Julien M. Canada Prenatal Nutrition Program, Part II: Review of Key Canadian Programs. Health Canada, January 1998, unpublished.

13. Higgins AC, Moxley JE, Pencharz PB, et al. Impact of the Higgins Nutrition Program on birth weight: A within-mother analysis. $J \mathrm{Am}$ Diet Assoc 1989;89:1097- 103

14. Buescher PA, Larson LC, Nelson MD, Lenihan AJ. Prenatal WIC participation can reduce low birthweight and newborn medical costs: A costbenefit analysis of WIC participation in North Carolina. J Am Diet Assoc 1993;93:163-66.

15. Mendelson R, Dollard D, Hall P, et al. The impact of the Healthiest Babies Possible program on maternal diet and pregnancy outcome in underweight and overweight clients. I Can Diet Assoc 1991;52(4):229-34

16. Poland ML, Giblin, PT, Walker JB, Hankin J. Effects of a home visiting program on prenatal care and birthweight: A case comparison study. J Community Health 1992;17:221-29.

17. Blondel B, Breart G. Home visits during pregnancy: Consequences on pregnancy outcome, use of health services, and women's situations. Semin Perinatol 1995;19:263-71.

18. Bradley PJ, Martin J. Issues inherent in measuring the impact of care coordination on pregnancy outcomes. Public Health Nurs 1996;13:276-83.

19. Kitzman H, Olds DL, Henderson CR, et al. Effect of prenatal and infancy home visitation by nurses on pregnancy outcomes, childhood injuries, and repeated childbearing. JAMA 1997;278:644-52.
20. Desjardins E. Healthiest Babies Possible: June 1994 - June 1996 Program Evaluation. Toronto Community Services Public Health, June 1997, unpublished.

21. MacMahon B, Pugh TF. Epidemiology Principles and Methods. Boston: Little, Brown and Company, 1970;235-37.

22. Blair C, Ramey CT, Hardin JM. Early intervention for low birthweight, premature infants: Participation and intellectual development. $A m J$ Ment Retard 1995;99(5):542-53.

23. Kramer M. Determinants of low birthweight: Methodological assessment and meta-analysis. Bull WHO 1987;65:663-737.

Received: April 27, 1998

Accepted: March 26, 1999

Éditorial, suite de la page 223

sortir de cette spirale pour s'orienter vers une amélioration de la santé et du bienêtre. La volonté de favoriser le développement sain des enfants n'a jamais été aussi forte dans notre pays qu'aujourd'hui, et de plus en plus tous les paliers de gouvernement consacrent des ressources pour traduire cette volonté dans la réalité. Au niveau provincial, on peut citer l'exemple notoire du Plan d'action pour les enfants de la Saskatchewan, province qui a mis au point un mécanisme de coordination intersectorielle des politiques et des services et qui fournit des ressources destinées aux interventions communautaires. ${ }^{17} \mathrm{Si}$ le soutien de tous les enfants et de leurs familles est susceptible de remporter l'adhésion générale et d'avoir des conséquences positives, des interventions ciblées sur les parents à risque élevé et sur leurs environnements sont également indispensables si nous voulons casser la chaîne de transmission des difficultés sociales d'une génération à l'autre. En outre, la notion des trajectoires de risque va dans le sens d'une intervention aussi précoce que possible (soit de la préconception à la naissance), tout en reconnaissant qu'il est possible de réorienter cette trajectoire avec des mesures indiquées à chaque étape de développement, sans préjudice des expositions antérieures de l'enfant. Les risques posés à la santé et au bien-être des enfants sont nombreux et divers, mais les possibilités de favoriser un développement sain ne le sont pas moins.

\section{REFERENCES}

1. Pole JD, Dodds LA. Maternal outcomes associated with weight change between pregnancies. Can J Public Health 1999;90(4):233-36.

2. Desjardins E, Hardwick D. How many visits by health professionals are needed to make a difference in low birthweight? A dose-response study of the Toronto Healthiest Babies Possible Program. Can J Public Health 1999;90(4):22428.

3. Wade TJ, Pevalin DJ, Brannigan A. The clustering of severe behavioural, health and educational deficits in Canadian children: Preliminary evidence from the National Longitudinal Survey of Children and Youth. Can I Public Health 1999;90(4):253-59.

4. Camiletti YA, Alder R. Learning needs as perceived by women less than or equal to 16 weeks pregnant. Can J Public Health 1999;90(4):22932.

5. Friel JK, Andrews WL, Edgecombe C, et al. Eighteen-month follow-up of infants fed evaporated milk formula. Can I Public Health 1999;90(4):240-43.

6. De Wals P, Trochet C, Pinsonneault L. Prevalence of neural tube defects in the Province of Quebec, 1992. Can JPublic Health 1999;90(4):237-39.
7. Barker DJP. Fetal origins of coronary heart disease. BMJ 1995;311:171-74.

8. Leon DA, Koupilova I, Lithell HO, et al. Failure to realise growth potential in utero and adult obesity in relation to blood pressure in 50 year old Swedish men. BMJ 1996;312:401-6.

9. Vagero D, Leon D. Ischaemic heart disease and low birthweight: a test of the fetal origins hypothesis from the Swedish Twin Registry. Lancet 1994;343:260-62.

10. Stein AD, Ravelli ACJ, Lumey LH. Famine, third-trimester pregnancy weight gain, and intrauterine growth: The Dutch Famine Birth Cohort. Hum Biol 1995;67:135-50.

11. Silverman BL, Rizzo T, Green OC, et al. Longterm prospective evaluation of offspring of diabetic mothers. Diabetes 1991;40(Suppl):121-25.

12. Hall AJ, Peckham CS. Infections in childhood and pregnancy as a cause of adult disease-methods and examples. Br Med Bull 1997;53:10-23.

13. Pettit DJ, Baird HR, Aleck KA, Bennett PH, Knowler WC. Excessive obesity in offspring of Pima Indian women with diabetes during pregnancy. N Engl J Med 1983;308:242-4S.

14. Pettit DJ, Knowler WC, Bennett PH, Aleck KA, Baird HR. Obesity in offspring of diabetic Pima Indian women despite normal birth weights. Diabetes Care 1987;10:76-80.

15. Streissguth AP, Moon-Jordan A, Clarren SK. Alcoholism in four patients with Fetal Alcohol Syndrome: Recommendations for treatment. Alcoholism Treatment Quarterly 1995;13:89-103.

16. Power C, Hertzman C. Social and biological pathways linking early life and adult disease. $\mathrm{Br}$ Med Bull 1997;53:210-21.

17. Government of Saskatchewan. Our Children, Our Future. Saskatchewan 's Action Plan for ChildrenFour Years Later. Regina, Saskatchewan, 1997. 